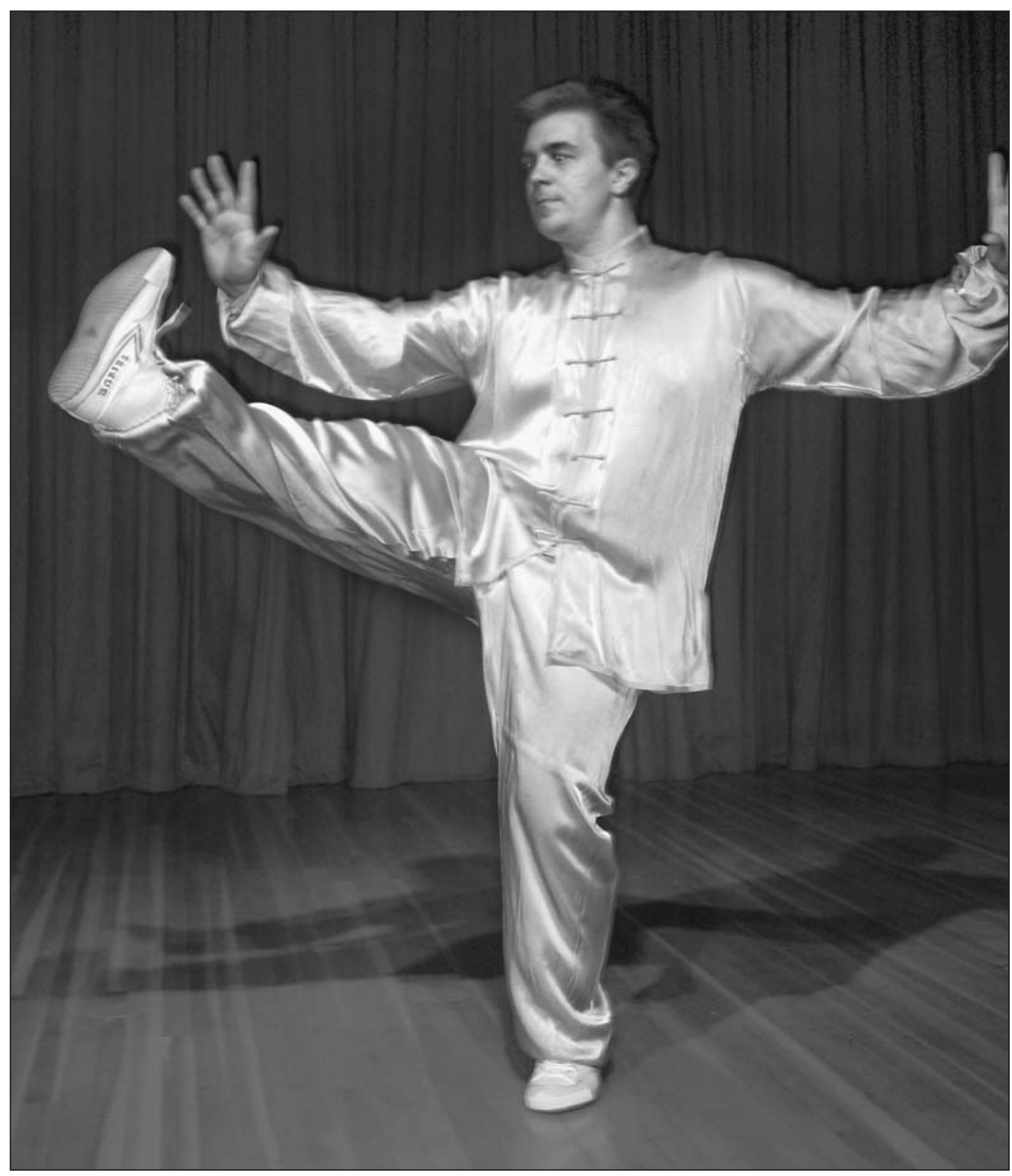

\title{
Resumen
}

Las caídas en las personas mayores pueden causar lesiones que llevan a una pérdida de independencia. La pérdida de control postural, de equilibrio, y los menores tiempos de reacción son factores importantes favorecedores de las caídas (Lajoie $\varepsilon$ Gallagher, 2004). Los programas de ejercicio tradicionales se centran en el estudio y tratamiento de estos factores (Tideiksaar, 1997); sin embargo, el miedo a la caída -otro fuerte elemento factor favorecedor- ha recibido una relativamente escasa atención en la literatura terapéutica (Maki et al., 199I). Existen indicadores de que existe una relación directa entre el miedo a caer y las caídas (Myers et al., 1996), así como una relación entre la evitación de actividades por miedo y las caídas (Delbaere et al., 2004). El taijiquan, una antigua arte marcial china convertida en método de ejercitación (Wolf et al., 2001), se ha mostrado como una práctica efectiva para mejorar el miedo a las caídas y a los antecedentes previos de caídas (Tsang et al., 2004). Como las terapias de exposición gradual, los practicantes de taijiquan logran lenta y progresivamente, en un entorno de calma, posiciones cada vez más difíciles que simulan situaciones potencialmente generadoras de miedo. En comparación con otros tratamientos mediante el ejercicio, tales como el entrenamiento del equilibrio computerizado, la educación y el ejercicio gradual, el taijiquan ha dado como resultado reducciones significativas del miedo a caer y de las caídas en sí mismas (McGibbon et al., 2005). En este trabajo se revisan las investigaciones existentes sobre el taijiquan y las caídas, defendiéndolo como una forma de terapia de exposición gradual para reducir el miedo a las caídas y las caídas en personas mayores. También se discutirán las consecuencias de estos trabajos y las futuras líneas de investigación. 


\title{
Miedo A CAER
}

\section{El TAIJIQUAN COMO FORMA DE TERAPIA DE EXPOSICIÓN GRADUAL EN VIVO}

\author{
Shane Kachur, R. Nicholas Carleton \& Gordon Asmundson
}

STS

\section{Introducción}

Las caídas en las personas mayores tienen unos enormes costes personales y de recursos (Tinetti \& Williams, 1997), llegando a menudo a una pérdida permanente de la funcionalidad y la independencia. Los esfuerzos preventivos para reducir las caídas se centran en sus causas, ya sean éstas internas o externas al individuo (Lajoie \& Gallagher, 2003). Los factores extrínsecos incluyen el calzado inadecuado, condiciones de vida inestables y las complicaciones derivadas de la climatología. Los factores intrínsecos incluyen las complicaciones neurológicas causadas por los medicamentos, síncopes, deterioro de la visión y debilidad. Definida como una reducción de las reservas fisiológicas y de las capacidades en las conductas (Wolf et al., 1996), la debilidad ha sido considerada durante largo tiempo como la causa más común de caídas en las personas mayores. Por el contrario, las investigaciones empíricas han identificado la pérdida de control postural, el equilibrio pobre y el miedo a las caídas como los tres elementos clave asociados a la predicción de caídas en poblaciones mayores (Lajoie \& Gallagher, 2003). Consecuentemente, el tratamiento tradicional para reducir las caídas ha consistido en programas de ejercicio centrados en reducir la debilidad e incrementar la fuerza, flexibilidad y equilibrio (Shumway-Cook, Gruber, Baldwin, \& Liao, 1997; Tideiksaar, 1997).

Se han utilizado mediciones del control postural y del equilibrio para determinar el riesgo de caída evaluando cómo las personas mayores controlan su centro de gravedad sobre su base de sustentación. Sin embargo, una elaboración cognitiva -el miedo a caer- puede afectar también a la ejecución física, reduciendo el equilibrio e incrementando la pérdida de control corporal (Tinetti, Medes de Leon, Doucette, \& Baker, 1994). Ciertamente, las mediciones del miedo a caer pueden ayudar a identificar a individuos que tienen riesgo de caer. Uno de los primeros intentos para identificar la relación entre la ejecución del equilibrio y el miedo a caer indicó que aquellos sujetos que decían tener miedo a las caídas mostraban resultados significativamente más pobres en control corporal en los test de equilibrio (Maki, Holiday, \& Topper, 1991). Desde entonces, se ha demostrado que el miedo a caer es un fuerte pronosticador de caídas en relación al control corporal y al equilibrio (Delbaere, Crombez, Vanderstraeten, Willems, \& Cambier, 2004; Lajoie \& Gallagher, 2003), y consecuentemente está siendo objeto de una atención adicional por parte de investigadores y personal médico. Más aún, la relación entre el miedo a caer y las caídas sugieren que los tratamientos que incluyan técnicas de reducción del miedo tendrán probablemente una mayor eficacia que los tratamientos que únicamente utilizan el ejercicio.
Todas las fotografías son courtesía de S. Kachur. 


\section{Investigaciones sobre el miedo a caer}

Las investigaciones han demostrado que el 30\% de las personas mayores de 65 años señalan que tienen un considerable miedo a caer, a pesar de que nunca hayan sufrido una caída (Hadjistavropolous \& Carpenter, 2006). En un intento por comprender cómo los indicadores psicológicos tales como el miedo a caer están relacionados con el equilibrio, Myers et al. (1996) evaluaron a un conjunto de 60 ancianos residentes de edades comprendidas entre los 65 y 95 años $(71,2 \%$ mujeres; $\mathrm{M}$. edad $=74.6$; $\mathrm{DE}=7,5$; véase la Tabla 1 para la terminología estadística). Se utilizaron dos autocuestionarios de respuesta si/no para medir el miedo a caer y la evitación de actividades -la Falls Efficacy Scale (FES; Tinetti, Richman, \& Powell, 1990)- y la Activities-specific Balance Confidence Scale (ABC; Powell \& Myers, 1995). Estos cuestionarios, junto con medidas posturográficas de equilibrio fueron examinados para identificar las relaciones entre varios indicadores psicológicos de la confianza en el equilibrio. Los grupos fueron separados según sus respuestas en la FES y después fueron comparados a partir de sus puntuaciones en la ABC. Los grupos con miedo a caer y la evitación de actividades puntuaron significativamente más bajo que el grupo sin miedo en la escala $\mathrm{ABC}(\mathrm{t}$ $=3.91, \mathrm{p}<.001$ y $\mathrm{t}=7.19, \mathrm{p}<.001$, respectivamente). La ABC también se correlacionó positivamente con las medidas de movilidad $(\mathrm{r}=.56, \mathrm{p}<.01)$. Posteriormente, Delbaere y cols. (2004) demostraron correlaciones negativas estadísticamente significativas $(r=-.49$, $\mathrm{p}<.01$ ) entre mediciones biomecánicas (i.e., fuerza física y control postural) y psicológicas (i.e., miedo a caer).

Un análisis similar entre el miedo a caer y el modo de andar (Chamberlin, Fulwider, Sanders, \& Medeiros, 2005) ha demostrado que los ancianos con miedo tienen unas características en su modo de andar significativamente diferentes -tales como menor velocidad, mayor anchura de paso, y una fase de apoyo con las dos piernas más larga- que el grupo control de ancianos sin miedo. Los cambios señalados en el modo de andar y las asociaciones con el miedo a caer, el comportamiento de evitación, y la ejecución física, apoyan que el miedo a caer es un factor importante en la ejecución física. El miedo y la evitación parecen tener alguna influencia en los mecanismos biológicos que controlan la locomoción y el equilibrio. Los miedos fuertes pueden tener influencias inmediatas, tales como un miedo repentino que provoca una caída. Los miedos sutiles pueden, con el tiempo, dar como resultado adaptaciones fisiológicas que contribuyen a un incremento de la debilidad.

Las dolencias somáticas incitadas (o exacerbadas) por el miedo y la evitación también han sido identificadas como un componente significativo en el desarrollo de dolores músculo-esqueléticos crónicos (Asmundson, Norton, \& Crombez, 2004). Abreviadamente, el miedo al dolor o al daño físico conduce a una ansiedad relacionada con el dolor que resulta en comportamientos de evitación y consiguientemente a un desuso y a una pérdida de condición (véase Figura 1). El ciclo de retroacción que se muestra en la Figura 1 muestra un

\section{TABLA I: Terminología estadística}

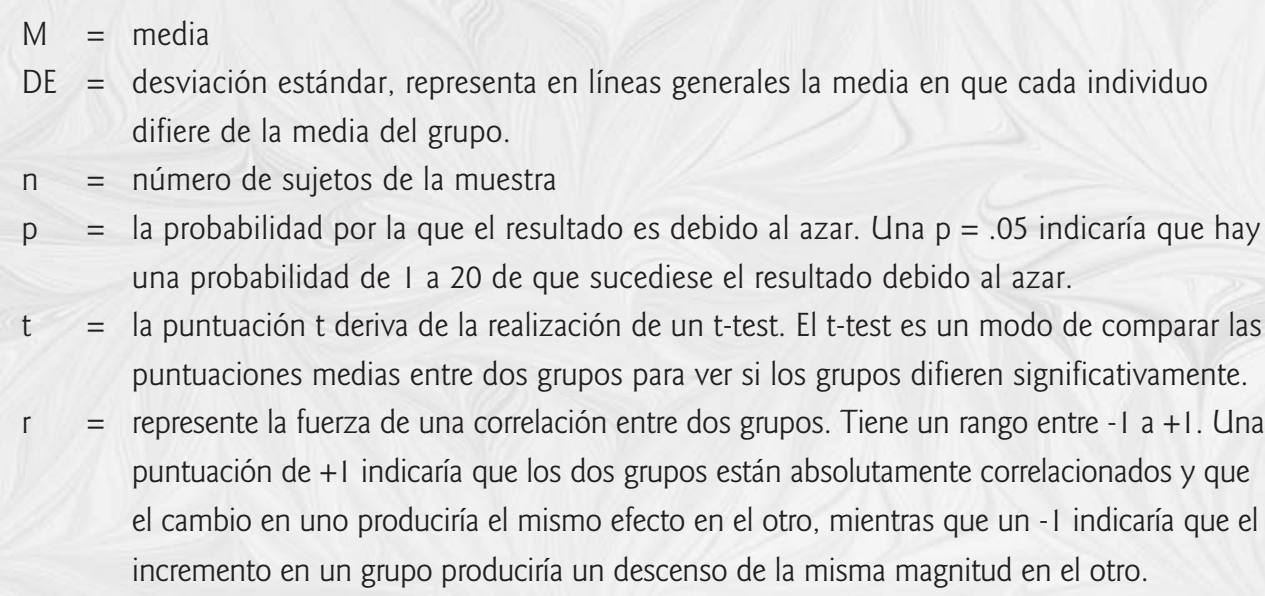




\section{FIGURA I: Modelo de dolor crónico de miedo-ansiedad-evitación}

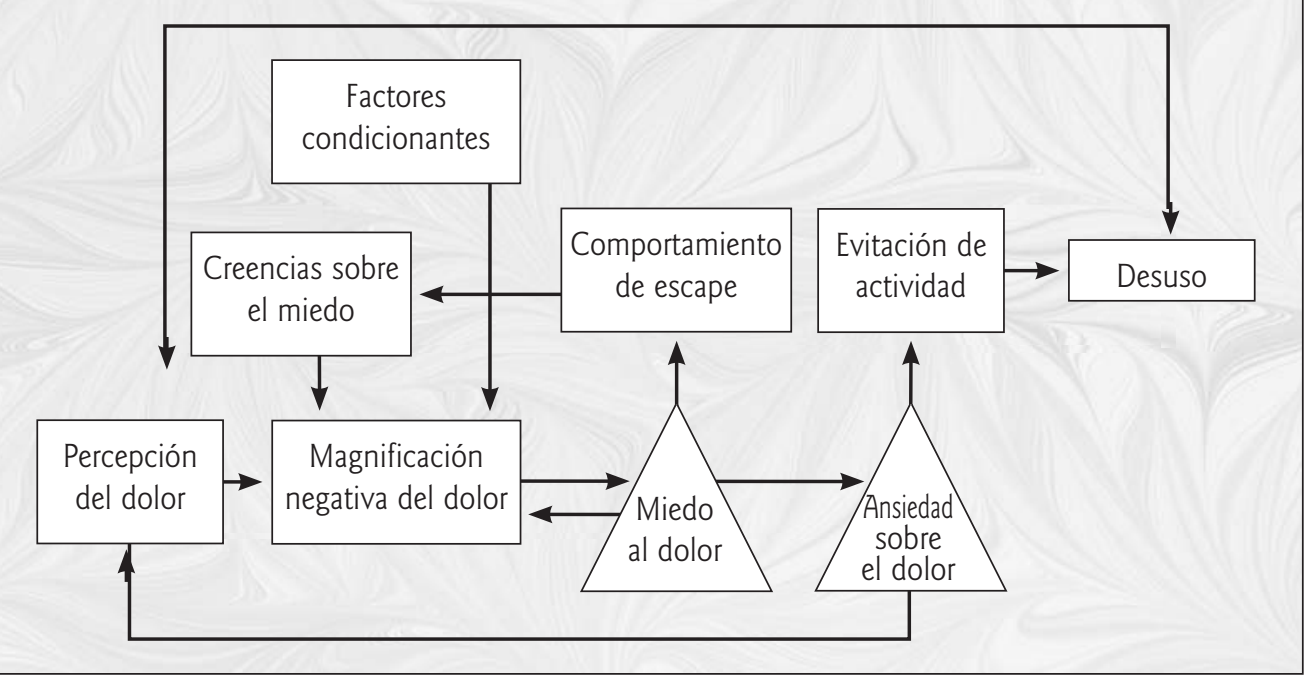

afianzamiento de las opiniones de miedo que sirve para mantener la evitación de actividad y la incapacidad. Basado en el modelo del miedo al dolor, puede proponerse otro modelo para la representación del miedo a caer en la evitación de actividades y las caídas (Figura 2).

\section{El papel del miedo a caer}

Las investigaciones existentes sugieren que el miedo a caer favorece las caídas de una o dos formas (Figura 2). En primer lugar, el miedo y la agitación resultante pueden dar como resultado cambios reales en la mecánica fisiológica de la marcha y en el control postural (Chamberlin et al., 2005). Estos cambios constituyen cambios medibles en la rigidez postural. La segunda forma está mediatizada por la evitación de ciertas actividades. La evitación de actividades conduce a pérdidas en la fuerza y en los rangos de movimiento, y favorece la debilidad, todo lo cual incrementa el riesgo de caídas (Delbaere et al., 2004). A través de estas vías, el miedo a caer puede actuar como un penetrante inductor de debilidad y de las caídas. El ciclo de retroacción que implica la rigidez postural y la evitación de actividad mantiene el miedo a caer, activando un círculo vicioso similar a los modelos de dolor crónico de miedoevitación (Asmundson et al., 2004). Por ejemplo, después de haber sufrido una caída, una persona tiene más riesgos de tener miedo a las caídas, lo cual lleva a la evitación, que puede llevar al desuso, a la rigidez postural y a un incremento del riesgo de caída.

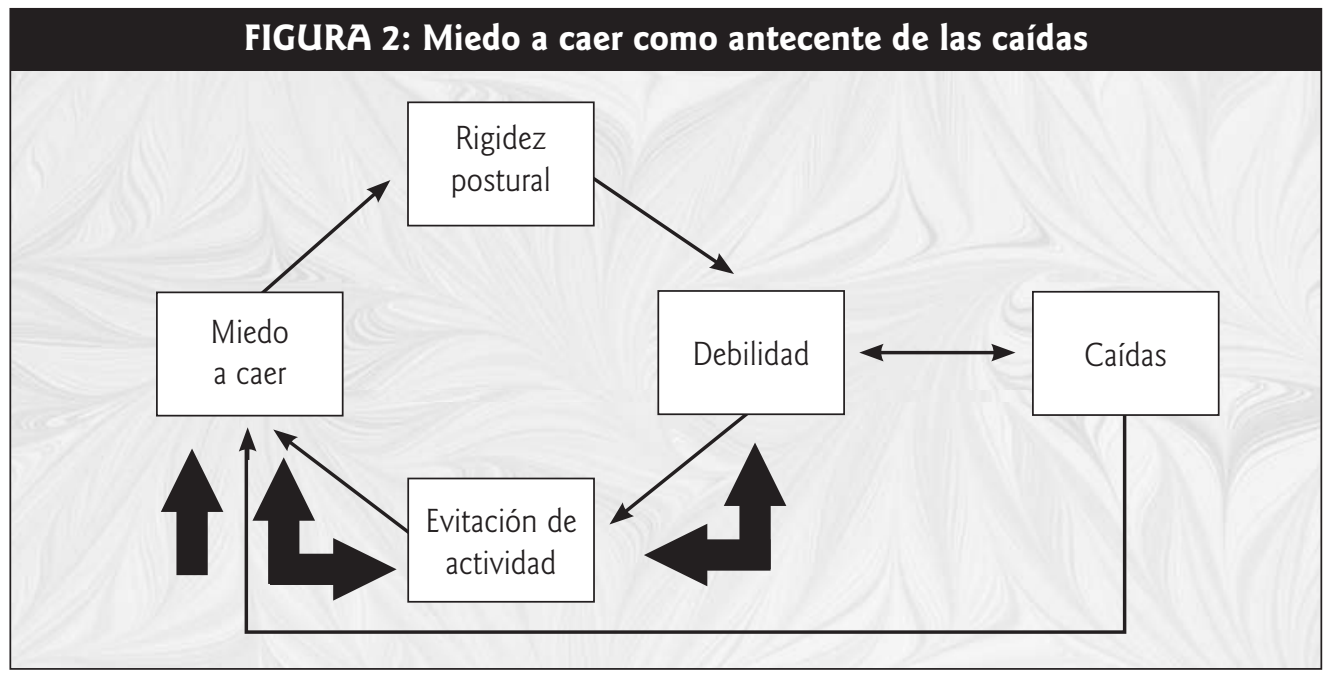

Revista de Artes Marciales Asiáticas $\diamond \quad$ Volumen 2 Número 2 (36-45) - 2007
Reelaborado y adaptado de Asmundson, Vlaeyen, \& Crombez (2004). Understanding and treating fear of pain, $p$. 15. Utilizado con permiso de la Oxford University Press 


\section{Tratamiento actual para el miedo a las caídas}

El creciente reconocimiento del papel que juegan el miedo y la evitación en las condiciones somáticas ha dado como resultado tratamientos especializados orientados psicológicamente, especialmente para aquellos que sufren dolores crónicos. El tratamiento efectivo del miedo y la evitación relacionada con el dolor crónico gira a menudo sobre un concepto denominado exposición gradual en vivo (en vida) (Vlaeyen, de Jong, Leeuw, \& Crombez, 2004). La exposición gradual en vivo implica la desensibilización sistemática a los estímulos que inducen el miedo a través de la exposición gradual a actividades generadoras de miedo en un entorno seguro. Se ha descrito sucintamente como "un método de tratamiento clave [en el cual] los individuos progresan a través de una serie de encuentros provocadores de ansiedad con estímulos fóbicos, mientras se utiliza la relajación como inhibidor recíproco de la creciente ansiedad" (Vlaeyen et al., 2004: 327). Es más probable que la confrontación de los estímulos generadores de miedo, y no el componente de relajación, sea el responsable primario de las reducciones de miedo. Negando la creencia de que se producirá algún daño en la actividad temida, la exposición gradual en vivo ha dado como resultado reducciones del miedo, dolor y la percepción de incapacidad, llevando a incrementos de la funcionalidad (Boersma et al., 2004; Vlaeyen, de Jong, Leeuw, \& Crombez, 2004).

\section{Taijiquan}

El Taijiquan es una antigua arte marcial china que ha evolucionado a método de ejercitación para la salud, resultando en mejoras de capacidad de movimiento, función cardio-respiratoria, equilibrio y postura (Wolf, Coogler, \& Xu, 1997). El taijiquan puede considerarse como una forma de exposición gradual en vivo. Lenta, pero progresivamente, el taijiquan expone a sus practicantes a posturas cada vez más dificultosas que estimulan situaciones que pueden provocar también miedo a las caídas. Clásicamente, las rutinas de taijiquan pueden tener más de 108 movimientos. Sin embargo, la mayoría de investigaciones utilizan formas modificadas que contienen únicamente 10-20 movimientos (Wayne et al., 2004). El taijiquan también promueve una actitud y una atmósfera de calma y relajación durante cada uno de los movimientos posturales. De este modo, abarca los dos componentes de exposición gradual y relajación propios de la exposición gradual en vivo. Como forma posible de exposición gradual en vivo, el taijiquan reduce el miedo a caer y mejora el equilibrio (Taggart, 2002; Wolf et al., 1996).

El taijiquan ha recibido una creciente atención científica como método de prevención de caídas en las personas mayores. Hasta ahora el centro de estos estudios ha sido los efectos del taijiquan en los sistemas de control físicos/neurológicos de la postura y del equilibrio (Haas et al., 2004; McGibbon et al., 2005; Tsang, Wong, Fu, \& Hui-Chan, 2004; Wayne et al., 2004; Wolf, Coogler, \& Xu, 1997). Estos estudios del equilibrio y la postura demostraron que, como estrategia de tratamiento, el taijiquan mejora el control postural y el equilibrio (Hass et al., 2004). Por otra parte, el taijiquan se muestra efectivo en la mejora del equilibrio y de la postura incluso para personas que sufren enfermedades vestibulares periféricas (McGibbon et al., 2005; Wayne et al., 2004).

El taijiquan puede contribuir a prevenir el debilitamiento. Se comparó una muestra de 20 practicantes de taijiquan $(50 \%$ mujeres; M. edad $=70.7 ; \mathrm{DE}=5.1)$ con una media de 7.2 años de práctica $(\mathrm{DE}=7.2)$ con un grupo control de 20 sujetos de edad similar $(60 \%$ mujeres; $\mathrm{M}$. edad $=67.8 ; \mathrm{DE}=4.5)$ y con un grupo de 20 estudiantes universitarios sanos $(40 \%$ mujeres; M. edad $=21.5 ; \mathrm{DE}=1.6)($ Tsang et al., 2004). Los grupos de practicantes de taijiquan y de estudiantes universitarios realizaron significativamente mejor los test computerizados de equilibrio que el grupo control de sujetos no practicantes. Esto sugiere que los practicantes de taijiquan (entre 65.6 y 75.8 años de edad) tienen el mismo control del equilibrio que individuos jóvenes y sanos. Este estudio y los estudios vestíbulo-posturales sugieren que el taijiquan mejora el equilibrio puesto que éste está gobernado por los sistemas neuromusculares. 
El taijiquan también ha sido comparado con los tradicionales métodos "Occidentales" para la mejora del equilibrio. Un ensayo clínico aleatorio controlado comparó el taijiquan, la terapia de equilibrio computerizada y la educación (Wolf et al., 1996). Se midieron variables biomédicas (fuerza, equilibrio y flexibilidad), funcionales (actividades diarias) y psicológicas (depresión y miedo a las caídas) en una muestra de 200 residentes ancianos [81.0\% de mujeres mayores de 70 años $(\mathrm{M}=$ 76.2)]. Los participantes fueron distribuidos aleatoriamente en tres grupos: Taiji $(n=72)$, entrenamiento del equilibrio computerizado $(\mathrm{n}=64)$, y clases de educación $(\mathrm{n}=64)$. El grupo de taijiquan practicó durante 15 semanas. Los análisis de mediciones repetidas mostraron que aquellos practicantes que habían realizado taijiquan mejoraron respecto a los otros dos grupos mediante una reducción estadísticamente significativa del miedo a caer $(\mathrm{p}<.05)$. No se encontraron diferencias significativas entre el grupo de taijiquan y el de terapia de equilibrio computerizada en las mediciones de equilibrio. Además, después de un mes de seguimiento del grupo de taijiquan éste tuvo 29 caídas, mientras que el de equilibrio computerizado tuvo 44 y el de educación 37. Se encontró que el taijiquan redujo el riesgo de caídas múltiples en un $47 \%$ durante este periodo de cuatro meses. También es digno de destacar que el coste por participante asociado con el grupo de clases de taijiquan fue más bajo que en entrenamiento computerizado del equilibrio, de carácter individual con un terapeuta físico. Taggart (2002) reprodujo los hallazgos de Wolf, encontrando un efecto similar en la práctica del taijiquan sobre el equilibrio, movilidad funcional y miedo a caer en mujeres mayores de 65 años. Después de tres meses de actividad normal los participantes realizaron clases de taijiquan dos veces por semana durante tres meses. Siguiendo en entrenamiento de taijiquan, se encontraron mejoras en equilibrio, movilidad funcional y miedo a las caídas. En relación a otras intervenciones alternativas, el taijiquan puede tener una mejor relación coste-efectividad y una mayor reducción del miedo a caer, mejorando el equilibrio, disminuyendo la ratio de caídas y atenuando el debilitamiento corporal (Tsang et al., 2005).

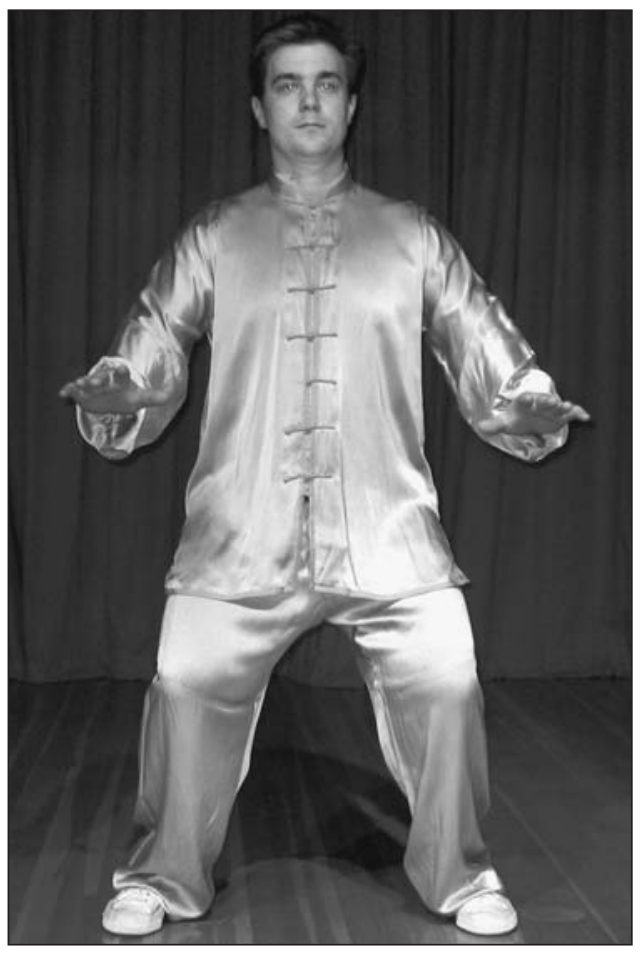

Fotografía I: Forma de inicio

Esta postura, con su amplia base de sustentación, tendría un factor de peligro bajo y puede hacerse más difícil añadiendo diferentes pesos o perturbaciones.

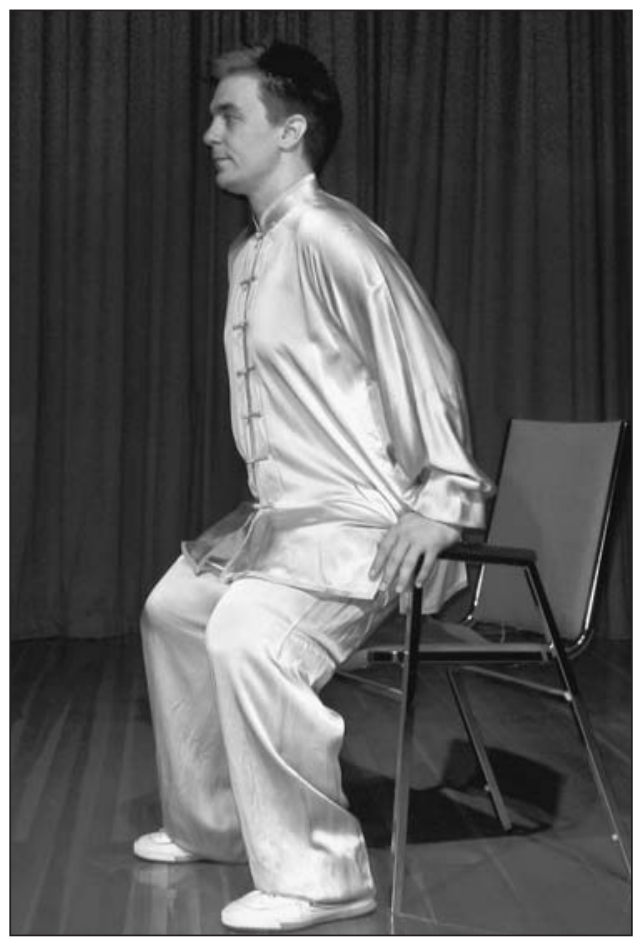

Fotografía 2: Forma funcional de inicio

Esta postura se asemeja a actividades funcionales clave tales como salir de la cama o levantarse de una silla. 


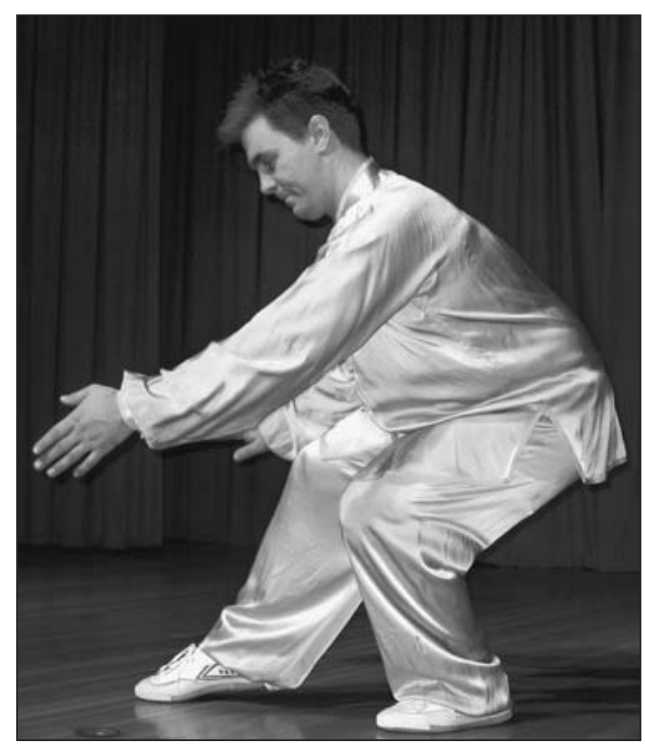

Fotografía 3: Aguja en el Fondo del Mar

Esta postura es más desafiante que la postura de inicio puesto que hay más peso en una pierna, más inclinación del tronco hacia el suelo y un mayor alcance.

\section{Fotografía 5: Permanecer} sobre Una Pierna

Progresando en dificultad, permanecer sobre una pierna presenta el mayor nivel de peligro ya que aumentan las exigencias de equilibrio. Es

importante afrontar los miedos a las caídas.

Esto puede hacerse reconociendo cualquier intento en la actividad y recompensando las ganancias, con el fin de

reducir el miedo y lograr la confianza. La diversión, centrándose en la relajación asociada al taijiquan, también puede ser beneficiosa.
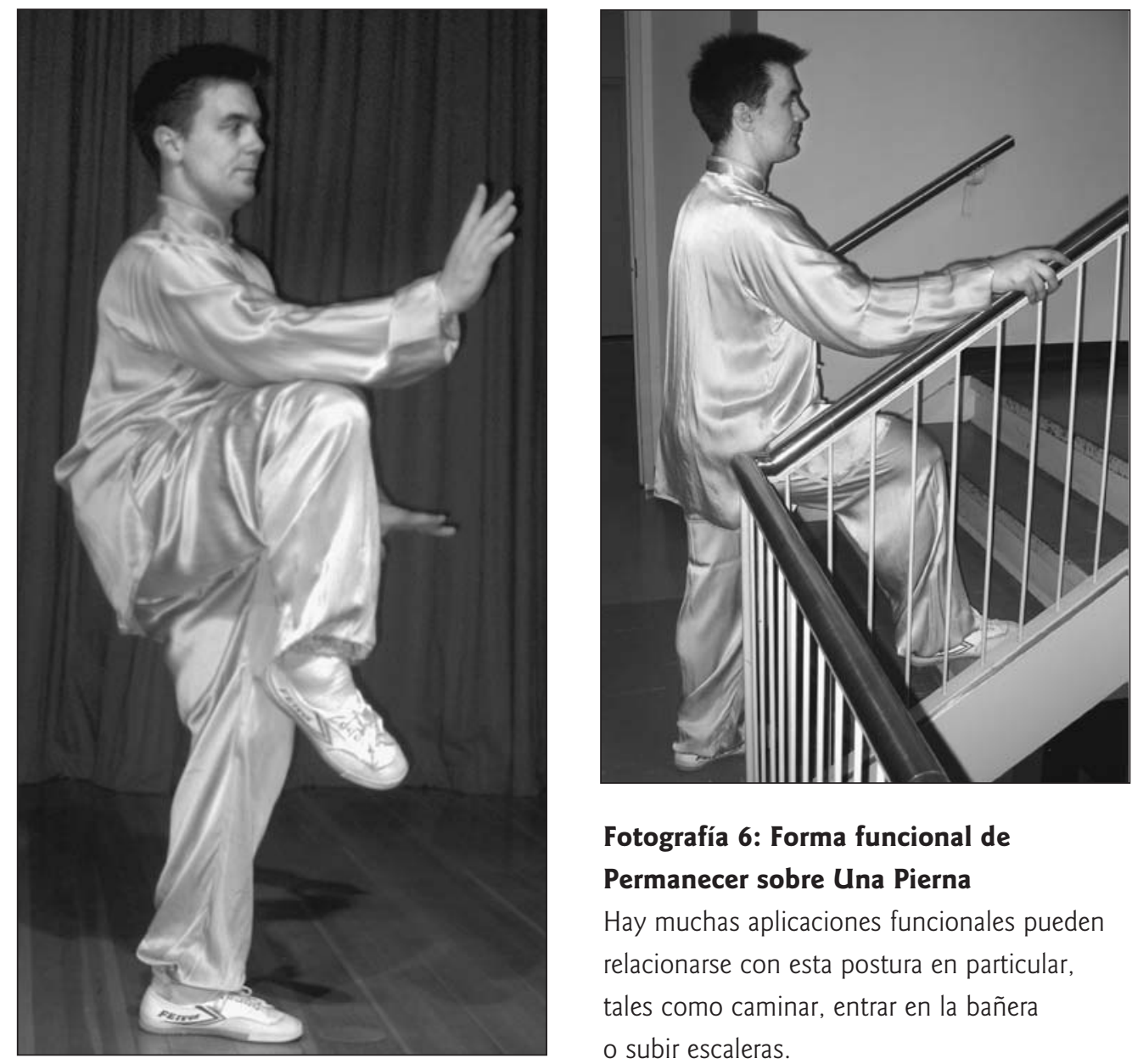

\section{Fotografía 6: Forma funcional de Permanecer sobre Una Pierna}

Hay muchas aplicaciones funcionales pueden relacionarse con esta postura en particular, tales como caminar, entrar en la bañera o subir escaleras. 
Además de los antecitados beneficios, el taijiquan también puede facilitar un régimen de ejercicio positivo socialmente reforzado. Si se utiliza la terapia del taijiquan previamente a haber sufrido una caída -o incluso mejor, antes de haber desarrollado cualquier tipo de miedo incapacitante a las caídas-, éstas pueden llegar a prevenirse en su mayoría. El concepto de taijiquan como forma de terapia de exposición gradual en vivo parece razonable y está apoyado periféricamente por investigaciones empíricas. Aún se necesitan investigaciones para evaluar esta posibilidad, y podemos citar los siguientes retos.

Deberían realizarse investigaciones específicas para llegar a decidir qué forma y perspectiva del taijiquan debería aplicarse. No existe una forma estandarizada de taijiquan y, más aún, existe una gran variabilidad en la experiencia de los instructores. Estas limitaciones metodológicas pueden hacer que sea difícil realizar generalizaciones de los hallazgos derivados de la investigación al conjunto de formas de instrucción. Existe, asimismo, un debate en los círculos de instructores de taijiquan sobre el papel que juegan las intenciones del estudiante en la obtención de mejoras de salud. Es decir, algunas escuelas de taijiquan opinan que los máximos beneficios para la salud sólo pueden adquirirse si el estudiante aprende el movimiento como arte marcial, más que como un ejercicio o terapia. Puede existir una resistencia sustancial por parte de los instructores para ajustar su pedagogía a propósitos terapéuticos. Sin embargo, ya hay escuelas de taiji que usan un modelo de danza, más que un modelo marcial, centrado en la coreografía y en la repetición sin preocuparse por los propósitos combativos del movimiento (Hong, 2006).

A pesar de los retos, el taijiquan parece tener un tremendo potencial para reducir las caídas. A este fin, deberían incorporarse cuatro principios basados en la exposición gradual en vivo en la enseñanza/práctica del taijiquan, con el objeto de maximizar los efectos de la exposición:

- Primero, asegúrese de que los estudiantes comprenden que el taijiquan no es peligroso. Esto debe realizarse verbalmente y a través de las demostraciones de los movimientos por parte del instructor, previamente a que los estudiantes los intenten o practiquen.

- Segundo, siempre que sea posible relacione los movimientos del Taijiquan con actividades de la vida diaria (véanse fotografías I a 6). La principal atención debería estar en los movimientos relacionados con las actividades de las que el estudiante ha manifestado sentir miedo. Debería existir asimismo una progresión comenzando por las actividades que dan menos miedo, incrementándose progresivamente a aquellas que más lo provocan.

- Tercero, los movimientos deben ser progresivos, comenzando por que provocan menos miedo e introduciendo progresivamente aquellos que más se temen según se vaya logrando éxito y adquiriendo confianza (véase fotografía 7).

- Cuarto, y último, enfréntese a las afirmaciones de los estudiantes que transmiten opiniones de que él o ella "es incapaz de", o "está en peligro de", mientras realiza cualquier actividad cotidiana. Esto debe culminar (después de un adecuado entrenamiento y práctica de taijiquan) explicando al estudiante que ya está realizando dicha actividad cotidiana durante la práctica de taijiquan. 
Los efectos de taijiquan, aplicado como una forma de exposición gradual en vivo, necesitan ser evaluados para asegurar sus beneficios potenciales sobre la salud, particularmente en la reducción de las caídas. Están documentados y se han comunicado a los profesionales de la salud interesados en la prevención del debilitamiento y de la enfermedad. Esperamos que este artículo influirá a los investigadores de la salud para estudiar el taijiquan como un método de prevención de caídas viable, atractivo, y con una buena relación coste-beneficio.

\section{N}

\section{BIBLIOGRAFÍA}

Asmundson, G., Norton, P., \& Vlaeyen, J. (2004). Fear-avoidance models of chronic pain: an overview. En G. Asmundson, J. Vlaeyen, \& G. Crombez (Eds.), Understanding and treating fear of pain. New York: Oxford University Press.

Boersma, K., Linton, S., Overmeer, T., Jansson, M., Vlaeyen, J., \& de Jong, J. (2002). Lowering fear-avoidance and enhancing function through exposure in vivo: $\mathrm{A}$ multiple baseline study across six patients with back pain. Pain, 108, 8-16.

Chamberlin, M., Fulwider, B., Sanders, S., \& Medeiros, J. (2005). Does fear of falling influence spatial and temporal gait parameters in elderly persons. The Journals of Gerontology. Series A, Biological sciences and medical sciences, 60, 1163-1167.

Delbaere, K., Crombez, G., Vanderstraeten, G., Willems, T., \& Cambier, D. (2004). Fear-related avoidance of activities, falls, and physical frailty. A prospective community-based cohort study. Age and Ageing, 33, 368-373.

Haas, C., Gregor, R., Waddell, D., Oliver, A. Smith, D., Flemming, R., \& Wolf, S. (2004). The influence of Tai Chi training on the centre of pressure trajectory during gait initiation in older adults. Archives of Physical Medicine and Rehabilitation, 85, 1593-1598.

Hadjistavropolous, T., \& Carpenter, M. (2006). Fear of pain and fear of falling in the elderly. Presentación en la Canadian Pain Society 2006 Annual Conference, Edmonton, 14 y 15 de junio. Edmonton, AB.

Hong, J. (2006). Chen style Taijiquan practical method (Z. H. Chen, Trad.). Edmonton, Canada: Hunyuantaiji press.

Lajole, Y., \& Galagher, S. (2003). Predicting falls within the elderly community: of postural sway, reaction time, the Berg balance scale, and the Activities-specific Balance Confidence Scale for comparing fallers and non-fallers. Archives of Physical Medicine and Rehabilitation, 85, 1593-1598.

Maki, B., Holliday, P., \& Topper, A. (1991). Fear of falling and postural performance in the elderly. Journal of Gerontology, 46, 123-131.

McGibbon, C., Krebs, D., Parker, S., Scarborough, D., Wayne, P., \& Wolf, S. (2005). Tai Chi and vestibular rehabilitation improve vestibulopathic gait via different neuromuscular mechanisms: preliminary report. BMC Neurology, 5, 1471-1483.

Miedo a caer: el taijiquan como forma de terapia... $\quad$ Shane Kachur, R. Nicholas Carleton \& Gordon Asmundson 
Meyers, A., Powell, L., Maki, B., Holliday, P., Brawley, L., \& Sherk, W. (1996). Psychological indicators of balance confidence: Relationship to actual and perceived abilities. Journal of Gerontology, 51, 37-43.

Powell, L., \& Myers, A. (1995). The activities-specific balance confidence (ABC) scale. Journal of Gerontology, 50, 28-34.

Shumway-Cook, A., Gruber, W., Baldwin M., \& Liao, S. (1997). The effect of multidimensional exercises and balance, mobility and fall risk in communitydwelling older adults. Physical Therapy, 77, 46-57.

TAggert, H. (2002). Effects of Tai Chi exercise on balance, functional mobility, and fear of falling among older women. Applied Nursing Research, 15, 235-242.

TideIKSAAR, R. (1997). Falling in the old age: Its prevention and management (2nd Ed). New York: Springer.

Tinetti, M., Medes de Leon, C., Doucette, J., \& Baker, D. (1994). Fear of falling and fall-related efficacy in relationship to functioning among community-living elders. Journal of Gerontological Medical Science, 49, 140-147.

Tinetti, M., Richman, D., \& Powell, L. (1990). Falls efficacy as a measure of fear of falling. Journal of Gerontological Psychologial Science, 48, 239-243.

TinetTi, M. \& Williams, C. (1997). Falls, injuries due to falls, and the risk of admission to a nursing home. The New England Journal of Medicine, 337, 1279. 1284.

Tsang, W. W., Wong, V. S., Fu, S. N., Hui-Chan, C. W. (2004). Tai Chi improves standing balance control under reduced or conflicting sensory conditions. Archives of Physical Medicine, 85, 129-137.

Vlaeyen, J., De Jong, J., Leeuw, M., \& Crombez, G. (2004). Fear reduction in chronic pain: graded exposure in vivo with behavioral experiments. En G. Asmundson, J. Vlaeyen, \& G. Crombez (Eds.), Understanding and treating fear of pain. New York: Oxford University Press.

Wayne, P., Krebs, D., Wolf, S., Gill-Body, K., Scarborough, D., McGibbon, C., \& KaptchuK, T. (2004). Can Tai Chi improve vestibular postural control? Archives of Physical Medicine and Rehabilitation, 85, 142- 152.

Wolf, S., Barnhart, H., Kutner, N., McNeely, E., Coogler, C., \& Xu, T. (1996). Reducing frailty and falls in older persons: An investigation of Tai Chi and computerized balance training. Journal of the American Geriatric Society, 44, 889-903.

Wolf, S., Coogler, C., \& Xu, T. (1997). Exploring the basis for Tai Chi Chuan as a therapeutic exercise approach. Archives of Physical Medicine and Rehabilitation, $85,886-892$

\section{NOTA}

Este trabajo ha estado apoyado en parte por el New Emerging Team Grant PTS—63186 del Canadian Institutes of Health Research (CIHR) Institute of Neurosciences, Mental Health and Addiction. El Dr. Asmundson está avalado por un Premio de Investigación del CIHR, y R. N. Carleton por un Premio de Investigación Doctoral del CIHR de Canadá. S. S. Kachur desea agradecer la ayuda y apoyo prestados por su profesor de taijiquan, el Maestro Nick Gracenin. La correspondencia relativa a este artículo debe dirigirse al Dr. Gordon J. G. Asmundson, Anxiety and Illness Behaviours Laboratory, University of Regina, Regina, Saskatchewan, S4S OA2. Tel: (306) 347-2415; Fax: (306) 585-4854; E-mail: gordon.asmundson@uregina.ca. 